\title{
Maschinenkonzept zur additiven Fertigung großdimensionierter Titan-Bauteile
}

Andreas Kalb, Florian M. Dambietz, Peter Hoffmann

In der vorliegenden Arbeit wird ein Maschinenkonzept präsentiert, welches für die Additive Fertigung von großvolumigen Titanbauteilen speziell entwickelt wurdet. Hierbei wird mit den Direct-Energy_Deposition Verfahren das Bauteil in einer separaten Inertgasatmosphäre erzeugt. Zur Führung der Prozesstechnik soll erstmals ein Roboter verwendet werden, der ebenfalls in dieser Atmosphäre verbaut ist. Dieser ist allerdings schwierigen Bedingungen ausgesetzt, da die Spannungsfestigkeit sowie die Isolationsschwelle in Argon im Vergleich zu Luft drastisch reduziert sind.

Keywords: Additive Fertigung, Direct-Energy Deposition, Inertgastechnik, Titanbauteile, Robotertechnik

\section{Einleitung}

Der Begriff 3D-Druck ist allgegenwärtig, egal ob im Freizeitbereich oder im industriellen Sektor. Dabei ist mit diesem Begriff primär nur ein Verfahren beschrieben welches in den Beriech der Additiven Fertigung gehört. Dieser Bereich wächst in den letzten Jahren drastisch an und bringt immer neue Ansätze zur Prozessführung und Maschinengestaltung hervor. Dadurch können neue Fertigungswege geschaffen werden, egal ob im Bereich Design, Materialoptimierung sowie bessere Festigkeitseigenschaften. Dies wird mittels Topologieoptimierung erzeugt und ermöglicht den Entwickler viel mehr Freiräume bei den Gestaltungsmöglichkeiten. Auf diese Punkte baut dieser Beitrag auf, da man nicht mehr aus einem ganzen Materialblock eine filigrane Kontur über zerspanende Prozesse erzeugen muss, sondern ein endkonturnahes Bauteil erzeugt, welches im Nachgang nicht oder nur minimal bearbeitet werden muss. Hierfür soll ein neuartiges Maschinenkonzept erarbeitet werden, das unter speziellen Atmosphären arbeiten kann und Bauteilgrößen größer 0,5 m² Grundfläche realisieren kann. Hierzu wird ein Träger für die Prozesstechnik benötigt wie ein Roboter zum Beispiel. Diese Handlingskomponenten sind allerdings in der speziellen Atmosphäre unter schwierigen Randbedienungen nur begrenzt einsetzbar. 


\section{Stand der Technik}

Durch die Additive Fertigung haben sich in der Herstellung von Bauteilen neue Bereiche ergeben. Hierbei ist wohl das beeindruckendste, dass die Bauteile direkt von ihrem digitalen Zwilling über Datenaufbereitung und -austausch in einer Maschine geschaffen werden können. Bei allen Fertigungsverfahren im Bereich Rapid Tooling erfolgt der Fertigungsprozess schichtweise [Poprawe: 2005, S. 225]. Durch diese generativen Verfahren können hochkomplexe, filigrane Bauteile erzeugt werden, die dennoch sehr starke Festigkeiten aufweisen [Richard: 2019, S. V].

Im weiteren Verlauf wird auf den aktuellen Stand des metallischen Rapid Manufacturing Bereich eingegangen. Hier sind die Fertigungsverfahren schon drastisch reduziert, da das Metall höher temperiert bearbeitet wird als Beispielsweise Kunststoffe. Hierbei ist das Temperaturfenster zwischen $600^{\circ} \mathrm{C}$ und $1400^{\circ} \mathrm{C}$ bei Metallen gängig. Die meist verbreiteten Verfahren sind Selectives Laser Sintern (SLS), Selectives Laser Melting (SLM) und Direct-Energy_Deposition (DED). [Poprawe: 2005, S231/Möller: 2017, S.17] Hierbei unterscheiden sich die ersten beide Verfahren gegenüber den letzten.

\section{Selektives Laser Sintern}

Das Selektiven Laser Sintern im Bereich von Metallen kann in zwei Bereiche aufgeteilt werden, einerseits in den Beriech Indirektes Selektives Laser Sintern und zum anderen in das direkte Selektive Laser Sintern von Metallen. Jedoch ist das Grundprinzip gleich. Das Material wird mit einer Rakel in einen fest definierten Arbeitsbereich dünnschichtig aufgetragen. Der Arbeitsbereich ist maximal $400 \times 400 \times 400 \mathrm{~mm}^{3}$ und die Aufbauraten werden mit einer Schichtstärke von ca. $100 \mu$ m aufgetragen. [Schmid: 2015, S. 4]. Danach wird dies mit einer Energiequelle bestrahlt und zum Aufschmelzen gebracht. Die Energiequelle ist meistens ein Laser. Diese könnten Faser-, Scheiben- oder CO2 Laser sein.

Beim indirekten SLS-Prozess ist das Metallpulver mit einer dünnen Schicht aus Polymeren überzogen, diese Polymerschichten verkleben beim Erhitzen. Dadurch wird die neue Schicht mit der alten verbunden. In einem darauffolgenden Erhitzungsprozess werden diese Polymersichten verdampft. Diesen Prozess nennt man Nachversintern. Die entstehenden Lücken im Rohling oder auch Grünling genannt werden mit einem Metallpulver, das einen niedrigeren Schmelzpunkt hat als der Rohling aufgefüllt. Dieser wird wiederrum in einen Erhitzungsprozess aufgeschmolzen. Die Schmelze verbindet sich dadurch mit dem Grünling und es ergibt sich so das fertige Bauteil. Dies hat allerdings schlechte Werkstoffeigenschaften, wie in Tabelle 1 gezeigt wird. [Poprawe: 2005, S. 227] 
Die zweite Variante des SLS Prozesses, das direkte Selektive Laser Sintern hat den großen Unterschied, dass das Metallpulver mit einem anderen Metallpulver vermischt bzw. das Pulver mit einem anderen Metall beschichtet wird, das eine niedrigere Schmelztemperatur besitz. Dadurch werden die Schichten durch das Aufschmelzen des Pulvers mit dem geringeren Schmelzpunkt aufgeschmolzen und so miteinander verbunden. Der Vorteil hier ist, dass keine Polymere als Verbindungsmittel genutzt werden, sondern Materialien die mit dem Grundwerkstoff verwand sind. Ein weiter Aspekt ist, dass bei richtigen Zusammensetzungen des Pulvers die Wärmenachbehandlung nicht benötigt wird. Diese Variante hat ähnliche Materialeigenschaften wie Sie beim indirekten SLS-Prozess geschaffen werden. Dies wird in der nachfolgenden Tabelle 1 verdeutlicht. Hierbei muss man die Werte des Pulvers auf Stahlbasis mit der Tabelle 1 vergleichen. Hierbei handelt es sich bei den SLS-Prozessen um Pulver auf Stahlbasis und bei dem SLM Bauteil um einen Werkzeugstahl X38CrMoV5-1. [Poprawe: 2005, S. 227-S. 237]]

\begin{tabular}{|l|l|l|l|}
\hline Eigenschaften & $\begin{array}{l}\text { Indirekt SLS Ver- } \\
\text { fahren }\end{array}$ & $\begin{array}{l}\text { Dirket SLS Verfah- } \\
\text { ren }\end{array}$ & SLM Verfahren \\
\hline Zugfestigkeit & $510 \mathrm{~N} / \mathrm{mm}^{2}$ & $500-550 \mathrm{~N} / \mathrm{mm}^{2}$ & $1720 \mathrm{~N} / \mathrm{mm}^{2}$ \\
\hline Bruchdehnung & $10 \%$ & $7 \%$ & $2,3 \%$ \\
\hline
\end{tabular}

Tabelle 1: Übersicht der Materialeigenschaften im Vergleich zu den

Pulverbettpassierenten Anlagen. [Poprawe: 2005, S. 227-S. 237]

\section{Selektives Laser Melting (SLM)}

Der SLM-Prozess ist eine Weiterentwicklung des SLS-Prozesses, dass am Fraunhofer Institut entwickelt wurde [Markenregistereintrag 30094322] Der Unterschied dieses Verfahren zu den anderen ist, dass es nur mit einem reinen Metallpulver betrieben wird und kein Bindemittel benötigt. Dadurch entfällt die zusätzliche Wärmebehandlung des Grünlings. Somit können reine Legierungen erzeugt werden, die auch z. B. im medizinischen Bereich Anwendung finden. Das erzeugen des Bauteiles ist nahezu identisch wie beim SLS-Prozess. Das Pulver wird wieder in einem fest definierten Bereich mit einer Rakel aufgetragen. Die Schichtdicken können variieren zwischen $30 \mu \mathrm{m}$ und $100 \mu \mathrm{m}$ [Poprawe: 2005, S. 111, S. 208]. Das Pulver wird mit einem Laser, der eine höhere Leistung als im SLS Prozess besitzt, Punktuell aufgeschmolzen. Hierbei wird das Pulverkorn komplett in ihre flüssige Werkstoffphase gebracht. Dadurch verbinden sich die Körner miteinander. Es wird sogar ein Teil der bestehenden Schicht darunter mit aufgeschmolzen. Dadurch erhält man hier nahezu identische Materialeigenschaft 
wie, wenn man z.B. ein gegossenes Bauteil zum Vergleich heranzieht. Dies ist in Tabelle 1 dargestellt. [Poprawe: 2005, S. 237]

\section{Direct-Energy Deposition (DED)}

Der DED Prozess unterscheidet sich zu den anderen Varianten dahingehend, dass er den Werkstoff für den Prozess nicht flächig aufträgt und dann dort aufschmilzt wo es benötigt wird, sondern er bringt das Material nur dort an, wo es auch benötigt wird. Dies kann über eine Drahtzufuhr oder über einen Pulverstrom realisiert und so an die Energiequelle geführt werden. Die Energie wird meist durch einen Elektronenstrahl, Laserstrahl oder Lichtbogen bereitgestellt. [Lukas: 2019]

Gegenüber den anderen Prozessen ergeben sich zwar einige Nachteile wie z.B. das keine Bohrungen oder große Hinterschnitte mit erzeugt werden können wie es im Pulverbett möglich ist. Auch die Genauigkeit ist im DED Verfahren schlechter, da mehr Material an die Schmelzzone herangeführt wird, als für das fertige Bauteil benötigt wird. Somit muss dieser Rohling immer nachgearbeitet werden.

Dieser Prozess hat seine Vorteile vor allem in der Bauteilgröße und den Auftragsraten. Die Bauteilgröße ist sehr variabel, hier kommt es vor allem auf die Prozessbedingungen an. Sofern keine spezielle Atmosphäre wie z. B. beim Titanschweißen benötigt wird, kann dieser Prozess große Dimensionen von mehreren Quadratmetern abdecken. Dies wird im Werkzeugbau durchs Laserauftragsschweißen schon seit mehreren Jahren praktiziert. [Lukas: 2019/Trumpf: 2016] Ein weiterer großer Vorteil des Prozesses ist, dass die Aufbauraten viel höher sind als in den anderen Prozessarten. Hierbei kann der Zuführdraht eine Stärke zwischen 1 und $3 \mathrm{~mm}$ besitzen und die Pulverpartikel sind zwischen 50 und 150 Mikrometer große Körnung aufweisen [Lukas: 2019]. Dadurch ist der Prozess vor allem für Reparaturarbeiten interessant. Dennoch wächst ein steigender Bedarf für dieses Verfahren an. Hierbei ist das Fertigen groß dimensionierter Bauteile relevant, die mittels des DED-Verfahrens Entkonturnah gefertigt werden und somit nur noch einer minimalen Bearbeitung unterzogen werden. Dies ist vor allem für sehr teure Werkstoffe interessant, ebenso wie für Materialen die sehr schlecht zerspanbar sind. Dadurch können Werkstoff-, Maschinen- und Werkzeugkosten signifikant gesenkt werden. [Trumpf]

\section{Erarbeitung eines neuen Maschinenkonzeptes}

Da der DED-Prozesstechnik noch ein enormes Potenzial hinsichtlich Werkstückgrößen und Werkstoffarten für das Bauteil besitzt, soll auf dieser Prozesstechnik aufgebaut werden. Damit eine große Vielfallt an Werkstoffen verarbeitet werden kann, soll der 
Prozess in einer separaten Atmosphäre stattfinden, die genau gesteuert werden kann. Dadurch wird der Arbeitsraum zwar von Beginn an eingeschränkt, aber diese Einschränkung ist sehr gut Größenabhängig kalkulierbar. Für diese Forschung wird sich auf einen Bauraum von $600 \mathrm{~mm} \times 600 \mathrm{~mm} \times 400 \mathrm{~mm}$ festgelegt. Dies wurde gewählt, dass man die ganze Prozesstechnik sowie den Arbeitsraum fest definieren kann und so genaue Bedingungen zum Bau feststehen.

Damit das Material an die Punkte kommt, wo es benötigt wird, muss eine Positioniereinheit integriert werden. An diese muss auch die Energiequelle befestigt werden, um große Arbeitsräume abdecken zu können. Für diesen Fall kommen meist CNC gesteuerte Portalanlagen Frage, ebenso wie roboterbasierte Systeme. Auf Basis von CNC-Anlagen gibt es bereits zwei Hersteller, die diese Prinzipien anbieten. Hierbei handelt es sich um die Französische Firma beAM und die amerikanische Firma Optomec. Allerdings ist ab einer gewissen Größe das Portal sehr kostenintensiv. Der Ansatz mit einem Industrieroboter würde die Bahngenauigkeit der CNC Anlagen zwar nicht erreichen, da das Bauteil aber nachgearbeitet werden muss und aufgrund der entsprechenden Spurbreite von 3-5mm ist eine Positionstreue im Bereich von Hundertstel-Millimetern nicht nötig. Für die beschriebene Entwicklung wird der Ansatz mit dem Roboter gewählt. Dies bringt zu gleich einige Herausforderungen mit sich, ob ein Roboter in einer separaten Atmosphäre arbeiten kann, die z. B. aus Argon 5.0 besteht.

Als Energiequelle wird in Anbetracht der Atmosphäre und der Prozesstechnik ein Laser ausgewählt. Mit einem Laser können zudem filigranere Bauteile erzeugt werden, da dieser durch Optische Elemente kleiner fokussiert werden kann und man somit mit dünnerem Draht oder sogar Pulver arbeiten kann. Darüber hinaus besitzt das fertige Bauteil weniger Eigenspannungen und während des Prozesses wird nicht so viel Wärme in das Werkstück mit Vorrichtung eingebracht. Ein Weiterer Vorteil ist, dass der Prozess flexibler ist. Hinzu kommt, dass der Auftragsscheißprozess besser gesteuert werden kann hinsichtlich Schmelzbadregulierung und flexibler auf unterschiedlichen Werkstoffen zum Verarbeiten eigegangen werden kann.

Bei dem benötigten Zusatzwerkstoff kann man, wie oben schon erwähnt, auf Draht oder Pulver zurückgreifen. Da man mit dem Pulverpassierten Prozess leichter auf Wandstärken und Werkstoffarten einflussnehmen kann, wird der Prozess auf PulverBasis aufgebaut. Ein weiter Punkt für diese Materialzuführung ist, dass in Verbindung mit dem Laser beim Auftragen nahezu identische Werkstoffeigenschaften vorhanden sind als bei gleichen Gussbauteilen. Zudem treten hier weniger Eigenspannungen im Werkstück auf. Dennoch muss der Wärmebehandlungszustand beachtet werden, da für die Nachbearbeitungen oder dem Endzustand das Bauteil durch Beispielsweise 
Spannungsarmglühen oder anderen Wärmebehandlungen den Bedürfnissen für den jeweiligen Einsatz angepasst werden kann oder muss.

Das Maschinenkonzept, dass in dem Layout dargestellt ist, isst wie Folgt aufgebaut. Die Anlage wird in einer eigenen Atmosphäre betrieben. Damit wird auf eine Glovebox zurückgegriffen. Der DED-Prozess basiert auf einem Laser-Prozess, der den Zusatzwerkstoff als Pulver zugeführt bekommt. Der Bearbeitungskopf wird in der Maschine per Roboter bewegt. Das Werkstück wird auf einer Substratplatte aufgeschweißt, diese soll in der Anlage reproduzierbar liegen. Hierzu wird das Bauteil mit deinem Transportsystem über eine Schleuse ein und ausgeschleust.

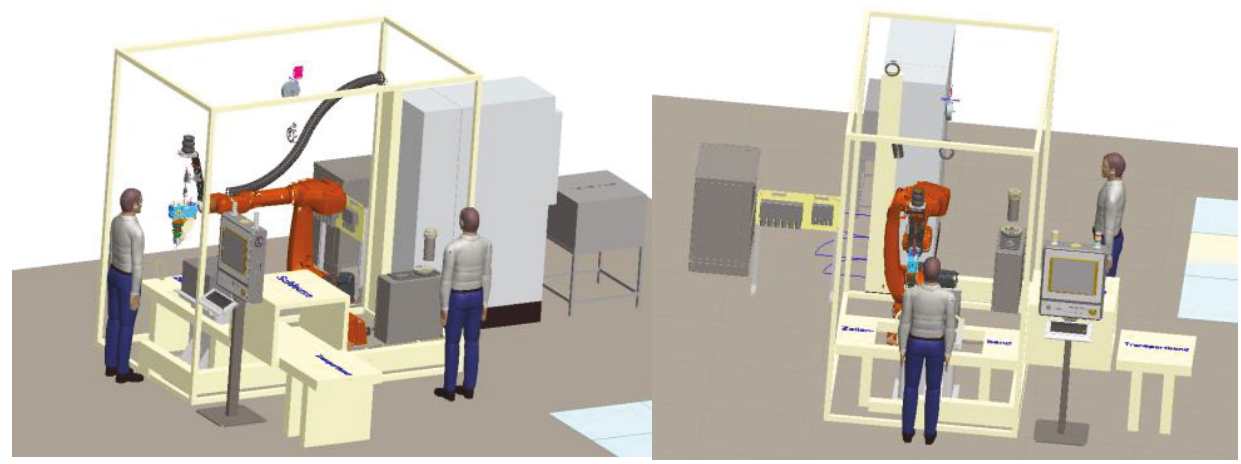

Abbildung 1: Layout der DED-Zelle

\section{Realisierung des Maschinenkonzepts}

Bei der Ausarbeitung des Maschinenkonzeptes muss auf einige Punkte strengstens geachtet werden. Das größte Augenmerk muss auf der Atmosphäre liegen, da hier die größten Anforderungen liegen. Hinzu kommt, dass noch keine Erfahrungen vorhanden sind, wie sich ein aktueller Roboter in einer Atmosphäre wie Agon verhält. Außerdem müssen die Elektronikkomponenten sowie Motoren geprüft werden, ob diese in dieser Atmosphäre arbeiten können bzw. wenn Sie arbeiten können, ob Sie ihr komplettes Potenzial abrufen oder nur zu einem gewissen Prozentsatz belastbar sind.

\section{Auslegung der Komponenten}

Für diese elektronischen Bauteile und Motoren werden Vorversuche im kleinen Stil gefahren. Dafür wird eine kleine Glovebox gebaut, damit die Motoren und kritische Bauteile unter Argon 5.0 über eine gewisse Zeit getestet werden können. Somit kann 
man diese Ergebnisse mit Versuchsergebnisse vergleichen, die unter Sandartbedienungen erzeugt wurden. Hierzu gehören die Motoren des Roboters, die Komponenten für den Pulverprozess und die Bauteile, die im Kopf verbaut sind. In der Abbildung 2 Ist der Versuchsaufbau dargestellt. Hier werden Motoren in einer kleinen Glovebox getestet. Diese wird mit Argon 5.0 geflutet. Ein Sensor überwacht den Versuch, dass eine reine Argon Umgebung über die Versuchsdauer gewährleistet ist.

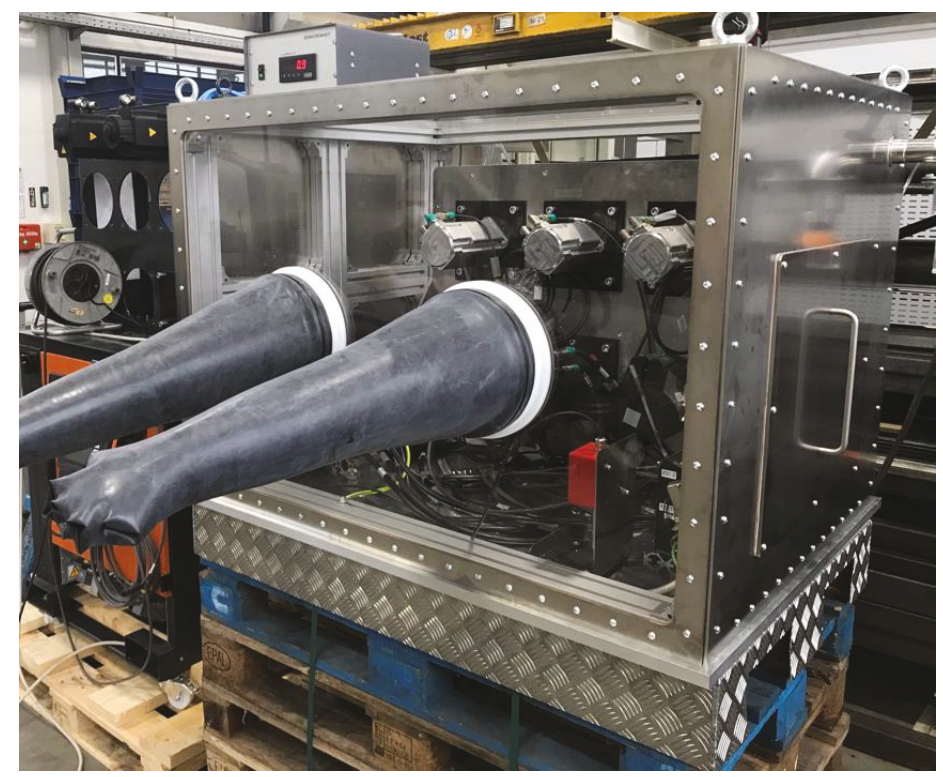

Abbildung 2: Versuchsaufbau in einer Glovebox

Ein wichtiges Auswahlkriterium sind auch die Maschinenkomponenten wie z. B. Dichtungen, Schläuche etc. die so gewählt werden, dass Sie die Atmosphäre nicht beeinträchtigen. Um zu verhindern, dass Materialen hier ausgasen und so die Atmosphäre zerstören. Dies soll ebenfalls in der Glovebox untersucht werden.

Ein weiterer Punkt sind die Medien, die sowohl in die Box eingeführt werden müssen als auch raus transportiert werden sollen. Es müssen Schnittstellen geschaffen werden, die gasdicht sind. 


\section{Auslegung der Größe der Anlage}

Da eine Glovebox mit Roboter sehr schnell sehr groß wird, muss hier eine Auslegung stattfinde, Hierzu wird ein Beschichtungskopf verwendet. Dieser muss für die Bedingungen angepasst werden, deckt aber sehr gut das Vorhaben ab. Da dieser festen Bedingungen wie z. B. Abmessungen, Gewicht, Schnittstellen etc. hat, kann damit die Robotergröße gewählt werden. Dadurch kann eine Simulation die Größe der Box für diesen Auftragsschweißprozess festlegen.

\section{Zusammenfassung und Ausblick}

Durch das neuartige Maschinenkonzept für die Additive Fertigung in Inertgas Technik hat sich ein großes Forschungs- und Entwicklungsprojekt ergeben. Da der Hauptbestandteil dieser Maschine mit einem Roboter und einen Laserpassierten Auftragsschweißprozess abgebildet werden soll. Dadurch ergeben sich neue Dimensionen in der Additive Fertigung, da ein Roboter große Bauräume abdecken kann. Hierzu muss nur der entsprechende Arbeitsraum hermetisch abgeriegelt sein.

In den nächsten Schritten müssen die Komponenten in Vorversuchen getestet und für die Argo Atmosphäre Qualifiziert werden. Damit diese in einem ersten Prototyp verbaut werden können. Hierzu kann es sein, dass Bauteile neu bzw. unter Berücksichtigung der Ergebnisse ausgelegt werden müssen. Wenn diese Ziele soweit erreicht sind, muss der DED-Prozess neu eingefahren und Qualifiziert werden.

\section{Literaturverzeichnis}

Poprwae Reinhart. (2005): Lasertechnik für die Fertigung: Grundlagen, Perspektiven und Beispiele für den innovativen Ingenieur. Berlin Heidelberg: Springer-Verlag.

Richard Hans Albert. (2019): Additive Fertigung von Bauteilen und Strukturen neue Erkenntnisse und Praxisbeispiele.Wiesbaden. Springer Fachmedien.

Möller M. et al. (2017): Additive Manufacturing of Large Scale Titanium Parts With Increased Productivit, Proceedings of the 6th International Workshop on Aircraft System Technologies AST. Hamburg.

Schmid Manfred. (2015): Additive Fertigung mit Selektivem Lasersintern (SLS) Prozess- und Werkstoffüberblick. Wiesbaden. Springer Fachmedien.

Lukas Johannes B. (2019): Ein Leitfaden zu Direct Energy Deposition (DED) im 3D-Druck. Hg. v. 3dnatives. Beitrag im Internet. https://www.3dnatives.com/de/ein-leitfaden-zu-direct-energy-deposition-ded-im-3d-druck, abgerufen am 05.03.2021.

Trumpf (2016): Laserauftragschweißen. Mit Materialauftrag neue Potenziale erschließen. Beitrag im Internet. Mit Materialauftrag neue Potenziale erschließen (trumpf.com) abgerufen am 05.03.2021. 
Markenregistereintrag 30094322.

Firma BeAM: BeAM Machines - Directed Energy Deposition (beam-machines.com), abgerufen am 04.03.2021.

Firma Optomec: Home - Optomec, abgerufen am 04.03.2021.

\section{Kontakt}

Andreas Kalb M.Eng.

ERLAS Erlanger Lasertechnik GmbH

Kraftwerkstrasse 26

D-91056 Erlangen

www.erlas.de

Prof. Dr.-Ing. Peter Hoffmann

Florian M. Dambietz M.Sc.

ERLAS Erlanger Lasertechnik GmbH

Kraftwerkstrasse 26

D-91056 Erlangen

www.erlas.de 